This item was submitted to Loughborough's Institutional Repository (https://dspace.lboro.ac.uk/) by the author and is made available under the following Creative Commons Licence conditions.

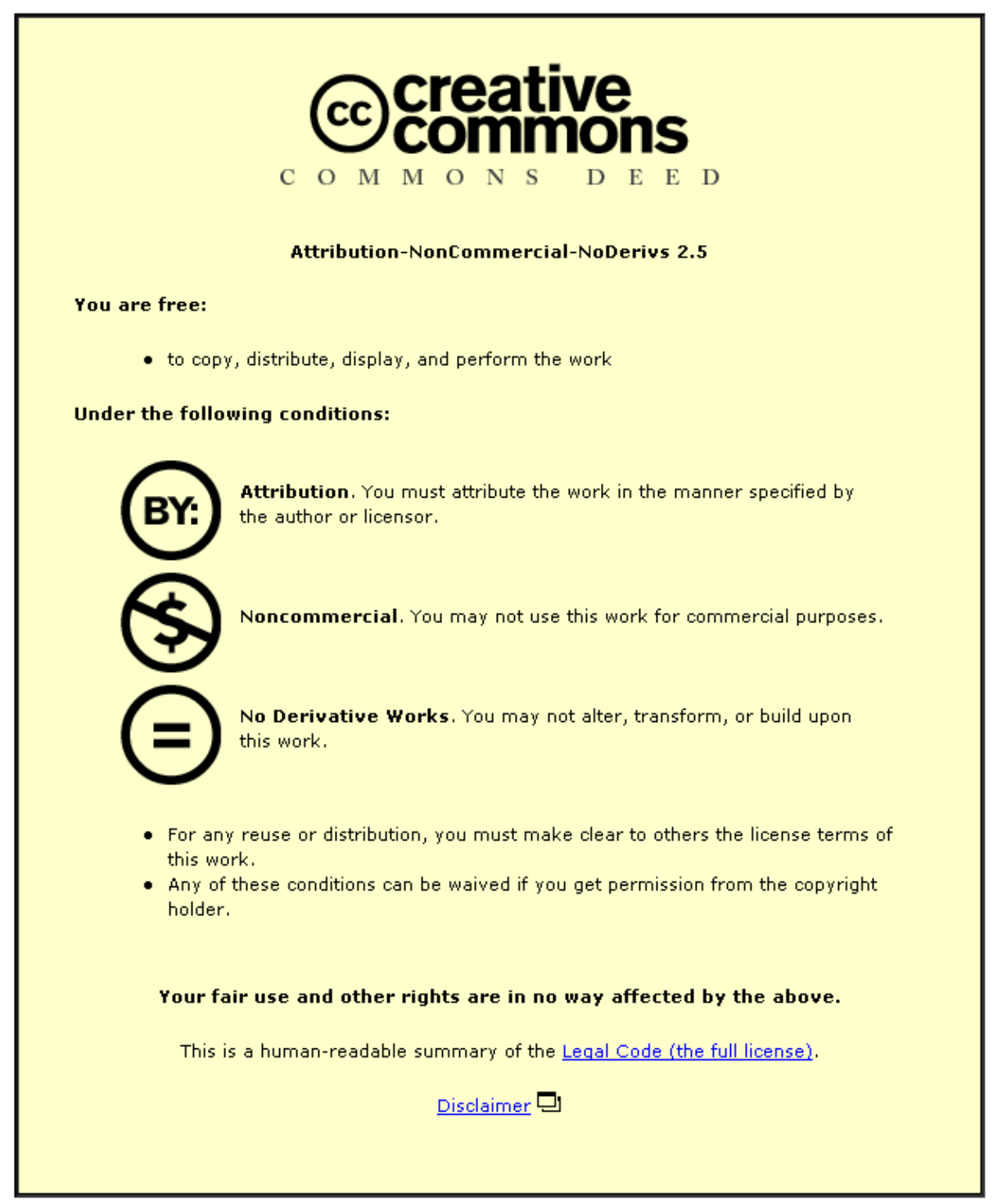

For the full text of this licence, please go to: http://creativecommons.org/licenses/by-nc-nd/2.5/ 


\title{
THE KNOWLEDGE MANAGEMENT TO LEARNING ORGANIZATION CONNECTION
}

\author{
Paul Chinowsky ${ }^{1}$ and Patricia Carrillo ${ }^{2}$
}

\begin{abstract}
The changes in the engineering-construction (E-C) industry of the $21^{\text {st }}$ century require organizations to take a more active role in developing knowledge management and learning organization initiatives. The need to both retain knowledge within the organization and focus on continuous human resource development throughout all levels of the organization is becoming a primary challenge throughout the industry. This paper addresses this challenge by focusing on the question of the link between knowledge management and learning organizations and how to transform an organization from a focus on knowledge management to a focus on developing a learning culture. Based on a series of studies by the PIs into the characteristics of both knowledge management and learning organizations, this paper outlines models of each of these concepts and introduces a bridge that details the level of knowledge management implementation that must be in place prior to an organization having the capacity to move to a learning focus. Additionally, the case studies conducted during the current study provide a basis for presenting potentially unsuccessful paths that may be selected by organizations during the implementation of a knowledge management to learning organization transition.
\end{abstract}

\section{KEYWORDS}

Construction, management, knowledge management, learning organizations

\footnotetext{
${ }^{1}$ Associate Professor, Department of Civil, Env, and Arch. Engineering, University of Colorado, Boulder, CO 80309-0428, (ph) 303-735-1063, paul.chinowsky@colorado.edu

${ }^{2}$ Professor, Department of Civil and Building Engineering, Loughborough University, Leics. LE11 3TU, England, (ph) 441509 222634, p.m.carrillo@lboro.ac.uk
} 


\section{Introduction}

The engineering-procurement-construction (EPC) industry of the $21^{\text {st }}$ century is undergoing significant changes as it addresses issues such as the introduction of advanced field and office technologies, the aging of the workforce, globalization, economic integration, and international partnering. These changes are initiating a challenge for the EPC industry in regards to how to educate personnel to appropriately respond to the rapid introduction of change within the industry. The foundation of this challenge focuses on how to both retain knowledge within the organization and establish continuous human resource development throughout all levels of the organization. At the present, the response to this challenge is focusing on the development of knowledge management programs where organizations emphasize the collection and managed distribution of knowledge within the organization. However, the scope of the changes within the construction industry requires EPC organizations to evolve one step further beyond knowledge management programs into learning organizations. In this evolution, organizations continously develop, capture, and pursue knowledge with the additional explicit purpose of continuously reviewing existing processes for opportunities to improve operations.

This move to a learning organization is a comprehensive transformation by an organization. However, the drivers for this move are well documented by researchers both within and outside the business domain (Goh 1998; McGill et.al. 1992; Stata 1989). Primary among these drivers is the emergence of the knowledge worker as the new model for an organization employee (Drucker 1993). The 1950s through the 1970s witnessed the strength of the manufacturing era where the production of goods dominated the economy. Within this economy, the production worker had primary importance. These individuals had the primary responsibility to assemble components into the finished assemblies that drove the production era. In contrast, today's economy with an emphasis on issues such as globalization and automation is 
moving toward the knowledge era where the manipulation and application of knowledge takes primacy over the production of components. In parallel with this transformation has been the emergence of the knowledge worker who is expected to understand how to apply knowledge in unique scenarios and with greater imagination and efficiency. Creativity has overtaken process as the foundation for successful solutions.

This emphasis on creativity and the application of organization knowledge places a spotlight on a critical divide between knowledge management and learning organizations that currently exists in the EPC industry. Specifically, the question of how to transform the organization from a focus on knowledge management to a focus on learning is the question addressed in this paper. Based on a series of studies by the authors into the characteristics of both knowledge management and learning organizations (Chinowsky and Molenaar 2005; Carrillo, et.al. 2004), this paper outlines models of each of these concepts and introduces a bridge that details the level of knowledge management implementation that must be in place prior to an organization having the capacity to move to a learning focus. The importance of this bridge to industry, and the relevance of this paper to the industry, being that relying on knowledge management is a static position for an organization that is not sustainable. Rather, the organization must put into place an active focus on learning to achieve long-term sustainable advantage.

\section{Background}

Although the concept of learning organizations may be new to EPC organizations, the concept is well-established in the management domain. Within this domain, three primary 
influences have merged to impact the development of learning organizations; learning, knowledge management, and organization systems.

\section{Learning}

The fundamental basis of establishing a learning culture is the requirement to enhance learning within the organization. Research within this domain divides learning into two distinct categories. Essentially, learning is categorized based on when and why it takes place and the effect that it has on those who are learning. The first type of learning can be thought of as incremental learning in which knowledge is gained in a piecewise manner as it becomes a necessity while the second is a dynamic process of continual learning in which knowledge is proactively sought out before it becomes a necessity.

Chris Argyris (Luthans et. al. 1995) describes learning as being either single-loop or double-loop. The fundamental difference between these two learning types is what they change. Single-loop learning changes a process in response to information from past experiences and focuses on the symptoms of problems. Double-loop learning uses symptoms only as indicators, shifting focus to the root causes of problems to change the underlying principles and theory behind a process. Essentially, single-loop learning takes place after a problem has occurred, making it a reactive process; whereas double-loop learning focuses on fixing processes before a problem occurs, making it a proactive process (Argyris 2000).

Similarly to Argyris, Senge categorizes learning into two separate categories: adaptive and generative. However, while Argyris' categorizations of learning center on what is changed during the learning process, Senge's categorizations focus more on when learning takes place. Adaptive learning is a company's method of reacting to a dynamic work environment so that a 
company making use of only adaptive learning remains stagnant in its knowledge until it is forced by some new experience to adjust. Contrastingly, generative learning enhances our ability to create (Senge 1990). In this sense, generative learning is inspired by the possibility of change in the future while adaptive learning is imposed by actual change in the present.

Although different in name, Senge's and Argyris' categorizations of learning are fundamentally the same. Single-loop learning is an adaptive process and double-loop learning is a generative process. Companies which are engaging in single-loop or adaptive learning processes are at a minimum addressing the need to change and adjust business practices (Kululanga et. al. 1999). Additionally, Nevis points out that engaging in generative learning should not negate the value of everyday incremental "fixes." (1995) Companies will inevitably face problems which were not addressed in any generative learning processes and will be forced to adapt if they hope to survive.

\section{Knowledge Management}

In today's dynamic business world, it is no longer enough for knowledge to be possessed at the individual level. New technologies and an abundance of competition require that knowledge be shared and utilized at an organizational level if a company hopes to survive. Hendricks and Vrien suggest that the knowledge assets possessed by a company create the possibility for sustainable competitive advantage (1999). This being the case, a company that manages knowledge effectively will have a better chance of long-term survival than those which lack in the same area (Nonaka and Takeuchi 1995). An effective knowledge management program adapts individual knowledge into information that can be readily used to the benefit of the organization as a whole. Aside from extracting and clarifying knowledge from the 
individual, knowledge management programs organize and provide structure to information so that it can be located and used effectively and conveniently.

The importance of knowledge management becomes transparent when considering the different forms which knowledge can take. Just as learning can be divided into the two distinct categories of single- and double-loop learning, knowledge can be classified as either tacit or explicit. Explicit knowledge is just what it claims to be - knowledge that has been explicitly explained, recorded, or documented (McInerney 2002). As explicit knowledge has, in some form, been communicated and can be formally documented, it can be shared relatively easily among individuals throughout an organization. The more difficult task of knowledge management then becomes that of managing tacit knowledge. Tacit knowledge may be considered intuitive knowledge guided by experience. This type of knowledge is based on experience, mental models and perspectives that are so deeply embedded in a person that the knowledge becomes second nature to an individual and, as such, is difficult to communicate. Central to the concept of knowledge management is either (a) putting individuals in touch with one another to share their tacit knowledge; and/or (b) transforming individuals' tacit knowledge into explicit knowledge, which can be used by the entire organization.

Failing to either share tacit knowledge or create explicit knowledge from tacit knowledge can result not only in losses to an organization but can also help to accelerate a competitor's advantage. In other words, by sharing and extracting an employee’s tacit knowledge a company multiplies the value which that employee adds to the company. If one company is able to share and/or extract tacit knowledge in this manner, it will excel above competitors who may have the same knowledge within their grasp but fail to share it on a company-wide basis. 


\section{Learning Organizations and the Construction Industry}

Despite a wealth of literature on learning organizations and organizational learning, a minimal amount of research exists on learning in the construction industry. In contrast, a significant amount of work has been published on the use of lessons-learned systems within construction organizations. This focus reflects the industry focus on single-loop or adaptive learning. Rather than focus on identifying issues before they arise, the construction industry is characterized by its focus on reacting to problems and striving to contain problem projects.

A research effort by Kululanga et. al. shows that UK contractors employ very few learning mechanisms as a means of creating new knowledge, and those that are employed are not developed to their full potential (1999). Fu et. al. suggests that the lack of intentional implementation of learning mechanisms or attempts at becoming learning organizations may be due to the high level of competitiveness within the construction industry (2002). As the construction industry is highly fragmented, many contractors focus only on short-term survival. This being the case, many companies do not have sufficient resources for developing a learning organization. Rather, the management approach to learning seems to emphasize a technologybased approach. Specifically, organizations that have identified knowledge as a corporate asset are emphasizing the development of database systems, Intranets, and other distributed systems that allow individuals to access data when it is required. As highlighted by recent research, this technology approach is now being extended beyond the bounds of the construction industry to include participants throughout the design-construction interface (Messner 2003).

Although this focus on technology provides construction organizations with an entry point into the learning domain, it does not meet the requirements of the learning organization. Specifically, a reactive based approach to learning does not drive the organization forward to 
continuously gain knowledge and update processes for improvement. Reflecting the need to move in this proactive direction, the current research effort puts forward the following learning organization definition based on work by others and a focus on the needs of the EPC industry. Specifically, the definition incorporates both the idea of active knowledge acquisition as well as application to serve the purpose of retaining competitive advantage through improved performance.

A learning organization is skilled at creating, acquiring, sharing, and applying knowledge, and embracing change and innovation at all levels, resulting in optimum performance and maximum competitive advantage.

\section{Knowledge Management and Learning Organization Models}

This section introduces two models to assess knowledge management maturing and learning maturity in organizations.

\section{STEPS Model}

The STEPS model helps organizations to structure and implement knowledge management and to benchmark their implementation efforts. The model was developed as part of a three-year UK-government funded project that investigated the relationship between knowledge management and business performance (Robinson et al., 2004). 
The five stages in the STEPS model (Start-up, Take-off, Expansion, Progressive, and Sustainability) reflect varying levels of KM maturity. Each level is characterized or associated with certain attributes and attribute dimensions. The key aspects of the model is shown in Figure 1, reflecting different emphasis at various stages.

The following descriptions provide an overview of the five maturity stages and the associated matrix levels that must be achieved.

Stage 1: Start-up: Organizations at this stage are the least advanced and are characterized by:

- An understanding of the concept of $\mathrm{KM}$, different perspectives and its practical implications;

- An appreciation of the benefits of KM, at least, in theory;

- Recognition of the potential of KM in building the value of knowledge assets for continuous improvement; and

- Establishing the need for KM and the willingness to share knowledge.

Figure 1: The STEPS Model

Stage 2: Take-off: Organizations have invested resources in $\mathrm{KM}$ and have an understanding of KM benefits and have a vision of what they wish to achieve. They are:

- Establishing the goals of KM; 
- Exploring strategic options. This could be demand driven (delivered in real time where and when it is needed) or supply driven (available in a central repository). The focus could be on people interactions (personalization) or documents or IT (codification);

- Developing a KM strategy with a working definition to facilitate consensus;

- Establishing leadership and identifying resources for consultancy and support;

- Identifying barriers and risks associated with the strategy and possible changes required; and

- Experimentation with KM on an ad hoc basis, localized or very small scale.

Stage 3: Expansion: Organizations at this level have been undertaking KM initiatives and wish to expand these either in terms of scope or depth. The Expansion stage is characterized by:

- Refining the KM strategy and linking KM to specific business objectives;

- Increasing the visibility of KM leadership, and the allocation of resources (budget, staff, IT infrastructure);

- Implementing a change management program to address barriers and risks identified;

- Implementing KM initiatives in a structured and coordinated way, and identifying appropriate KM tools to support specific initiatives;

- Increasing the scale of KM initiatives to other business units, projects and offices; and

- Introducing performance measures to evaluate $\mathrm{KM}$ and communicate the benefits of knowledge assets. 
Stage 4: Progressive: Organizations at this level focus on the improvement of their KM initiatives by measuring their impact. The Progressive stage is characterized by:

- Integrating KM activities to strategic measurement frameworks such as the Balanced Scorecard to monitor and evaluate knowledge assets;

- Establishing evaluation criteria and targets for measuring the impact on knowledge assets and justifying KM initiatives;

- Introducing reward and incentive schemes to strengthen KM activities; and

- Increased visibility and communication of the benefits from most KM activities.

Stage 5: Sustainability: At the Sustainabilty stage, KM becomes institutionalized and is characterized by:

- $\quad \mathrm{KM}$ becoming linked to all business objectives;

- KM practices diffused in the entire organization;

- KM becoming embedded in organizational culture, employees' behavior, business processes and product development; and

- Widespread reporting on the performance of knowledge assets underpinning corporate sustainability.

Learning Organizations Model

The learning organizations maturity model presented here is based on work conducted by the Construction Industry Institute on Learning Organizations in construction (Chinowsky and Molenaar 2005). The model was developed to provide 
construction organizations with a framework that identifies a path forward for establishing a learning organization culture. From this perspective, the matrix framework illustrated in Figure 2 was developed to facilitate the development process.

\section{Entity of Learning}

The entities of learning across the top of the matrix provide a reference point to the primary learning groups found within an organization. Each of these groups is dependent on each other to facilitate the exchange, development, and evaluation of knowledge.

- Organization — the overall corporate entity including all levels of management and staff personnel.

- Community — the entity that represents a group of individuals who are engaged in similar technical activities, commonly referred to as a community of practice (COP).

- Individual — the cornerstone of the learning organization since it is the individual that is responsible for actively seeking new knowledge and in turn disseminating knowledge to the organization.

\section{Characteristics}

The overall definition of a learning organization is further specified based on a series of five characteristics as follows:

- Leadership — the ability to lead the organization toward implementation of a learning organization. 
- Processes and infrastructure — the combination of the management processes and the technical infrastructure required to implement the learning organization vision within an organization.

- Communication - the interaction between both COPs and individuals within the organization that facilitates the free sharing of knowledge at all times and at all levels.

- Education — commitment by both management and employees to continuous education opportunities is a foundation of the learning organization concept and the key to bringing new knowledge into the organization.

- Culture - the final characteristic of learning organizations is the development of a culture that supports, promotes, and rewards learning as a vital part of organization enhancement.

\section{The Matrix as a Maturity Model}

The evolution to a learning organization is defined as a five-level approach with each level representing a stage of development towards a mature learning organization concept. Each level is defined as an organization having completed the implementation of specific matrix concepts (the boxes are indicated in dark fill) or the organization is actively addressing specific concepts (the light fill boxes). As an organization achieves the complete range of implementation levels for each box, the organization is considered to have achieved that level of maturity.

Level 0: just beginning the transformation to a learning organization concept.

Considered the base layer where all organizations begin. Although some activity may 
be occurring in individual matrix boxes, the transition to a Level 1 organization is still occurring.

Level 1: focused on establishing the leadership required to move toward a learning organization starting from an individual level (represented by the matrix completion evaluations). Additionally, the organization will begin addressing the processes and infrastructure that will be required to implement the knowledge sharing concept that key to a learning organization.

Level 2: leadership transformation completed and individual/community levels of process and infrastructure developed. Additionally, organization is actively addressing the communication aspects of learning and the initial stages of education and culture change at the individual and community levels. Actively moving toward and supporting a new focus on knowledge sharing and open communication.

Level 3: organization-wide processes to support learning fully implemented, with a new focus on the learning culture at the individual and community levels. Learning is no longer viewed as a necessary human resources requirement, but as an integral part of an individual's job and career.

Level 4: almost mature. Communication and sharing are now part of the corporate culture. Leadership is championing learning throughout the organization and at all levels. Additionally, the culture is strongly focused on learning at the community and individual levels throughout the organization.

Level 5: maturity in the learning organization model achieved. All boxes are now filled in within the model. Each level has adopted the complete range of learning 
organization characteristics, and the learning organization culture now characterizes the organization.

\section{Bridging the Models}

As outlined in the Introduction, the focus of the current research effort is to identify a bridge between the knowledge management and learning organization models. Specifically, to identify at what stage of the STEPS model can an organization bridge over to the LO model and successfully pursue a learning organization culture. To facilitate this identification process, the authors undertook a series of four case studies with organizations that were previously identified by the authors as having active efforts in knowledge management and learning. The focus of these case studies was to identify three items: 1) an evaluation of the organization on the STEPS model, 2) an evaluation of the organization on the LO model, and 3) the identification of the barriers and strategies that existed for the organization to advance its current level of learning implementation. Based on this identification, the focus of the research shifted to the main emphasis of identifying the bridge between the two models.

\section{Case Studies}

The case study process focused on conducting in-depth interviews with individuals in organizations that were actively pursuing knowledge management initiatives and were at least in the early stages of pursuing a learning organization culture. Four engineering-construction organizations were involved, based on their documented development of knowledge management and learning initiatives. The companies selected were each based in the UK but each had significant international operations in different 
parts of the world. This component was an important factor in selecting the organizations due to the greater need for sharing and disseminating knowledge across geographically distributed offices. This section highlights the methodology used in the case study process and the results obtained during the interviews. After completing the interviews, the team was able to analyze the results to propose the knowledge management - learning organization bridge.

\section{Methodology}

The first step in the case study process was to select the organizations that would be included in the process. Two key requirements were put in place for the selection process; 1) the organization must have a documented focus on pursuing knowledge management initiatives and have previously stated a desire to pursue a learning organization culture, and 2) the organization must have a record of pursuing knowledge management initiatives over a period of time that was sufficient to obtain insights into the barriers and opportunities available to this pursuit. As a secondary consideration, the team consciously decided to select organizations that were actively involved in international operations which required the organization to address geographic, cultural, and divisional differences. Based on this criterion and the contacts that were available to team members, four UK-based organizations were included in the final interview population as follows:

1. Company A is an international consulting firm focusing on the key areas of infrastructure and transportation. The company has a specific individual in charge of coordinating knowledge management activities. 
2. Company B is an international firm that has a dual focus on construction of major facilities as well as a property development division. The company has a Knowledge manager in charge of knowledge management initiatives internationally.

3. Company $\mathrm{C}$ is an international consulting firm focusing on public infrastructure projects and management of infrastructure in conjunction with public officials. Company $\mathrm{C}$ has a director of learning to focus specifically with public highway agency owners.

4. Company $\mathrm{D}$ is an international engineering-construction firm that constructs major facilities of all types in all regions of the globe. Company D has a team of individuals responsible for knowledge management learning initiatives.

Once the final organizations were selected, interviews were arranged with the one or two individuals who had the responsibility of overseeing the knowledge management process for the entire organization. In some cases this oversight was direct with each of the operating units and in some cases this focused on managing individuals who were in charge of the knowledge management process at respective units. The methodology employed for the case study process was a semi-structured interview process. In this process, the authors interviewed the learning organization team at the office of the interviewee. The topics used in the interview process are illustrated in Figure 3. As illustrated by the topics, the focus of the interview was to allow the organization as much opportunity as possible to elaborate on topics and provide input on their experiences in establishing a learning organization culture. The following sections provide highlights of the responses to the questions during the interview process. 


\section{Knowledge Sharing}

A fundamental component of both knowledge management and learning is the concept of knowledge sharing. In this context, knowledge sharing encourages the collection and dissemination of knowledge throughout the organization. Each of the four companies selected for the follow-up studies has an established history of knowledge management activities that emphasized knowledge sharing. At this point in time, only one of the companies believes it is successfully achieving knowledge sharing at an acceptable level. Within this organization, the combination of engineering collaboration and demand from the client is a driving influence in supporting knowledge sharing activities. The common issues stated with the other organizations focused on barriers from divisions, geographic distribution, or having the "will, but not the implementation". In these organizations, the common thread is a focus on project delivery over organizational collaboration. Although this is believed to be a reaction to client demands, this short-term perspective is having noticeable ramifications on long-term knowledge sharing initiatives. Specifically, the absence of focus on long-term initiatives is resulting in a reduction in resources focused on knowledge sharing.

\section{Communities of Practice}

Within any large organization, individuals can begin to feel isolated and lose a sense of "team" due to the feeling that they are not making a significant contribution to the organization or to a project. Communities of Practice (COPs) are one tool used to counter this feeling by providing individuals with a community of individuals, each of whom have similar technical or managerial responsibilities. In terms of knowledge management and learning, these 
communities serve a critical purpose in terms of promoting and supporting knowledge development, sharing, and use both within the individuals that belong to the community and to the greater organization. In the organizations that were interviewed for this study, the designfocused organizations, Companies A and C, each had formal COPs that provided strong support for their knowledge management activities. However, in the construction-focused organizations, these COPs were less evident due to a stronger focus on project teams rather than technical responsibilities. Similar to the knowledge sharing topic, the focus on delivering the project in these latter organizations was overshadowing the focus on organization collaboration. The result of this lack of focus on communities was a much lower emphasis on groups of individuals assessing and promoting new ideas within the organization.

\section{Leadership Support}

The third area of focus in the study was leadership support. This issue is critical to developing a learning culture since learning and knowledge management are organization-wide issues that require support beyond an individual project or group. The four organizations interviewed in this process each have leadership teams that are aware of the importance of these activities based on past commitments to knowledge management initiatives. However, the continuation of this commitment is mixed at best. Company $\mathrm{A}$ is witnessing the strongest continued support with top management providing active support, establishing a formal policy on knowledge sharing, encouraging employee participation through rewards, and committing substantial resources to the learning effort. Although not as focused as Company A, Company C also is receiving strong support for developing new ideas, although this is influenced by individual clients and projects. Where new ideas are client encouraged, the company is 
committing resources and has established a formal policy encouraging knowledge sharing. In contrast to these efforts, Companies B and D are witnessing a decrease in leadership support for knowledge initiatives. Specifically, these organizations are experiencing inconsistency in support as management determines the value of these initiatives to the overall organization. The result being that the individuals given the responsibility to oversee these efforts are less inclined to undertake ambitious efforts and instead focus on smaller initiatives requiring less support and resources.

Barriers

Similar to any initiative that requires management support and involves organization change, knowledge management and learning initiatives are experiencing barriers within each of the organizations studied. In this category, there is similarity between each of the companies in the barriers that are being encountered. Specifically, the common barriers are as follows:

- Value - a great difficulty in implementing knowledge management and learning initiatives is the task of providing direct financial results for the efforts. Unfortunately, learning is an indirect benefit to the organization that is difficult to quantify. The result of this difficulty is that business cases for these activities are not as strong as competing initiatives and subsequently receive less focus than initiatives that demonstrate direct financial gain.

- Project-focus - as discussed previously, knowledge sharing and learning are organization-wide initiatives that emphasize organization improvement over project delivery. This presents a barrier for many of these initiatives as the engineeringconstruction culture emphasizes project delivery as the central operating premise. 
Therefore, implementing a knowledge initiative requires the organization to alter traditional perspectives away from project delivery to long-term organization improvement.

- Culture - the implementation of any new initiative requires change. The implementation of change is difficult in any environment, but can be especially difficult in engineeringconstruction organizations where tradition and conservative practices are normal operating procedures. This barrier is being reached in each of the organizations in different forms. For example, in Company D, the culture difference is seen as a generation gap with newer generation employees more willing to share knowledge. In contrast, Company A the culture difference is geographical with different geographic locations being more willing to share knowledge than others with different cultural backgrounds. In each case the barrier returns to a central theme of requiring people to change traditional norms and procedures. Each of the organizations agreed that this is a major barrier to long-term success of these initiatives.

- Information Systems - knowledge management and learning are not information technology issues. However, information technology is essential to providing the infrastructure that allows organization employees to share and access knowledge. It is in this role that information systems emerged as a barrier for several of the organizations contacted in this study. For example, Company A faces the difficulty of implementing knowledge sharing across an organization that has an information system that was developed to focus on individual business sectors rather than overall organization requirements. In this manner, information systems may not provide the final answer for 
establishing learning strategies, but they can erect the barriers that prevent the strategies from being implemented.

- Stability - an underlying issue that organizations in the EPC industry have traditionally encountered is the lack of stability in the workforce. The perceived transient nature of construction work that results in personnel leaving companies to join other organizations that have preferred projects leads some organization to believe that an investment in knowledge management is difficult to justify. Specifically, the perception is that an investment in learning is lost when turnover rates are high and the knowledge leaves for another organization. This is a consistent remark that appears in interviews in different forms. This challenge is one that requires an underlying perception change within the industry and represents a topic by itself in terms of barriers to industry personnel investment.

\section{Strategies}

The identification of barriers leads directly to the identification of strategies to overcome these barriers. Although each of the organizations studied in this effort have adopted different strategies in different barrier situations, several common strategies have emerged for moving the learning initiatives forward. Common strategies identified in the study include:

- Face-to-Face Communications - providing individuals with a greater understanding of why new initiatives are being undertaken is a key strategy to overcoming change barriers. Both Companies A and D place extensive emphasis on working with individuals as a key strategy to enhancing knowledge sharing. In each case, communication of goals, 
objectives and long-term plans are considered the core of successfully obtaining the “buy-in” from organization personnel.

- Benefit Demonstration - the demonstration of bottom-line results was identified previously as a primary barrier to learning initiatives. Responding directly to this barrier, the interviewees expressed a focus on turning this barrier into a strategy. Specifically, as expressed by Company B, demonstrating a benefit either tangible or intangible on which a business case can be developed is an essential step in the initiative process. Although this strategy may be the most difficult to formulate, it remains at the forefront for every organization due to the belief that demonstrating tangible benefits is the most successful path to obtaining leadership support.

- Corporate Mandate - an official mandate from senior management will always get the attention of organization employees. That is the reasoning behind the strategy of using top executives to mandate knowledge initiatives. As outlined by Company $\mathrm{C}$, the use of an executive mandate results in an immediate impact as employees are energized to respond to the new organization objective. Capitalizing on this immediate impact is the focus of this strategy as champions of the knowledge initiatives are able to put in place demonstration tests that are aimed at producing rapid success stories. However, this focus may be short-lived if commensurate resources and follow-up are not put forward by corporate management.

In summary, the case studies presented in this section served a primary purpose of providing foundational evidence for the knowledge-learning link described in the next section. As outlined in the focus areas above, the relationship between initiatives, barriers and strategies is highly focused on the ability of the organization to demonstrate benefits from implementing 
learning activities. The next section builds on this requirement by outlining the link between knowledge management efforts and the transition to a learning organization culture.

\section{The KM-Learning Link}

The authors have previously established the potential benefits of pursuing a knowledge management or learning culture strategy. However, as stated at the beginning of this paper, the question of how an organization moves from a knowledge management initiative to a proactive learning initiative is a gap in current engineering and construction research. In an effort to bridge this gap, the authors studied the responses from the case studies described above to develop a proposed bridge between the two knowledge concepts. Specifically, the responses from established knowledge-focused organizations provided the foundational insight required to determine when and how an organization can successfully make the transition from a reactive approach to knowledge to a proactive approach.

The proposed bridge between knowledge management and learning initiatives is illustrated in Figure 4. As illustrated in this figure, the connection between the two concepts is based on the knowledge management STEPS and learning organization maturity models. The left side of the figure illustrates the STEPS model with each of the five levels from start-up through sustainability. In addition to these five steps, a preparation step has been added for those organizations just beginning to approach knowledge management and a continuation step has been added for organizations who are continuing to refine knowledge management practices beyond the maturity stage. Similarly, the learning maturity model is illustrated on the right side of the diagram. In this illustration, the five stages of maturity are illustrated vertically from 
establishing to maturing. Once again, the preparation and continuation stages have been added at the beginning and end of the process.

The beginning of the link between these two models resides in the STEPS knowledge management model. Since learning cannot occur without an active pursuit and management of knowledge, the establishment of a knowledge management initiative is essential to the eventual movement to a learning culture. As illustrated in Figure 4, since knowledge management must come first in the process, the beginning of the STEPS model is pictured with a lower first step than the learning maturity model. In the process of establishing a knowledge management focus, an organization will move through the first and second steps with a focus on an individual project or group. Since learning is an organization activity that requires sharing among all segments of the organization, these first stages in the knowledge management process are not conducive to a sustained learning initiative. Therefore, the first two stages of the knowledge management are considered preparatory for learning rather than the launching points for a learning initiative.

In contrast to the first two stages of knowledge management, the third stage of the STEPS model, Expansion, focuses on the organization transitioning from a project-focused knowledge management initiative to an organization-based initiative. Specifically, this stage witnesses the organization expanding knowledge management beyond a single project to multiple projects or multiple groups within the organization. This Expansion stage is critical to the eventual transition to a learning culture since it is at this stage that knowledge sharing among individuals outside of a constant working group begins to appear.

Once the Expansion stage has been achieved in the knowledge management model, the organization is ready to expand the knowledge initiative in two directions, mature knowledge 
management and establishment of learning. In terms of the former, the organization should continue to refine and expand its knowledge management efforts to achieve a mature knowledge management implementation. However, at this stage, the organization is ready to initiate a transition to a learning culture. Specifically, the existence of a knowledge management infrastructure and an awareness of the need to share knowledge are the essential precursors to initiating a learning initiative. Thus, as illustrated in Figure 4, the bridge between knowledge management and learning is established between the Expansion stage in STEPS and the establishing stage in the learning maturity model.

Although the existence of a knowledge management initiative in the Expansion stage does not ensure a successful transition to a learning initiative, it is proposed that this is an essential requirement for a successful transition. Given that this requirement is in place, an organization can transition to a learning initiative by establishing a focus on leadership and communications within the learning context. Since the organization is already emphasizing a knowledge focus, this transition should be a natural evolution. The primary difference being that the organization must now begin to emphasize proactive knowledge acquisition and an examination of existing practices to determine the potential for enhancements and changes in standard practices. Once this change in focus occurs, the organization can begin an active move toward establishing a learning culture by progressing through the learning maturity model.

\section{Unsuccessful Model Relationships}

The proposed link between knowledge management and learning presents a potential roadmap for organizations moving toward a learning culture. However, as documented by the authors during this study and previous case studies, organizations do not always follow a 
preferred path in a roadmap. Specifically, the existence of the knowledge management and learning models in a single roadmap provide opportunities for organizations to embark on alternative paths between and through the models. Some of these alternatives may result in similar endpoints, but others can result in serious limitations and long-term delays for organizations pursuing a learning culture. A few of these negative alternatives are discussed here as follows.

- Perpetual Management - The first unsuccessful venture an organization can undertake is attempting to adopt a fully sustainable knowledge management process prior to embarking on a learning initiative. In this scenario, the organization perpetually strives to refine its knowledge management system with the belief that the perfect knowledge storage and retrieval system is the foundation for learning. In reality, this approach results in an organization finding it difficult to ever reach the first level of the maturity learning model since it is reluctant to champion a change in its knowledge management course. Specifically, the organization remains mired in an endless effort to achieve knowledge management perfection without having the ability to adapt to a dynamic learning process.

- Insufficient Preparation - The second unsuccessful venture an organization can take into learning is to attempt to move from the take-off stage of knowledge management directly into the learning process. The difficulty with this combination is the lack of organization focus put in place prior to moving from knowledge management to learning. Rather than moving from a project focus to an organization focus and then to a learning focus, these organizations attempt to move from a project focus to a learning focus with no intermediate organization emphasis. As detailed earlier, the cornerstone of learning is a 
focus on organization knowledge sharing. Organizations that attempt this direct transition fail to put in place this essential step and find themselves compounding the difficulty of establishing an organization-based rather than a project-focused learning initiative.

- Loss of Focus - The final unsuccessful combination outlined here is the loss of focus that can occur between knowledge management and learning initiatives. Specifically, the authors found a common thread within organizations where a successful knowledge management initiative approaching the progressive stage was unable to match that success in the transition to a learning initiative. The common thread in these organizations was a reduction in resources directed toward the learning initiative. In these organizations, management would believe that the success in knowledge management translated to a reduction in resource requirements to continue success. The move to maturity in these efforts was interpreted as a move toward self-sufficiency. In reality, these efforts were only entering a resource dependent phase as they now required resources to communicate the need for learning and initiate new learning initiatives. This contradiction between reality and interpretation was found to be a direct precursor to reducing the effectiveness of learning initiatives and an indication that organization personnel would soon lose their focus toward establishing a learning culture.

Although these are only a few of the potential relationships that can result in negative transitions between knowledge management and learning, they represent common difficulties encountered by study participants. This commonality reinforces the need for organizations to 
follow the proposed roadmap to minimize the potential for delays in the transition between the two models.

\section{Conclusion}

The topics of knowledge management and learning organizations have each received considerable attention in recent years, both in the academic and professional communities. However, the link between these two subjects in terms of progressing from a knowledge management strategy to a learning organization initiative is less apparent. This paper has attempted to fill this gap by providing a link between the two topics based on a combination of the STEPS and Learning Maturity models previously developed by the authors. As outlined in the paper, the progression from knowledge management to a learning culture is dependent on the successful initiation of a knowledge management strategy. Once this strategy is in place, the expansion of knowledge management beyond a single project or group is the preparatory step to a learning initiative. At this stage, an organization can cross the bridge to the learning model and commence a focus on establishing the leadership required to initiate a learning culture.

Although organizations may choose to follow this progression using different path combinations, these same organizations should understand that it is possible to fail at crossing the bridge between the two concepts. The case study process in this study provided clear evidence that organizations who do not retain a focus on supporting these initiatives beyond the initial knowledge management stage will ultimately fail to realize the full potential of a learning initiative. Specifically, issues such as loss of employee support, reductions in knowledge sharing and generation, and reductions in organization communication are only a few of the 
ramifications that were noted in the interviews due to reductions in leadership support during the transition from knowledge management to learning organization initiatives.

In contrast to these negative results, the organizations that are successfully negotiating the transition between the two initiatives are obtaining notable results. In these cases, the organizations are using both initiatives to mutually strengthen both paths. The continued focus on knowledge management is assisting the learning effort by expanding the focus on organization communication, an essential element of expanding the learning culture. Similarly, the expansion of the learning infrastructure is assisting the knowledge management effort by providing greater opportunities for the organization employees to store, access, and share knowledge. This symbiotic relationship is not only advancing both initiatives, but it is also providing the tangible results demanded by senior management.

In summary, the path from a successful knowledge management initiative to a successful learning organization initiative has many options for success. This paper provides one path based on essential requirements for making the link between the two concepts. The next step for an organization is to evaluate where it currently stands in the process and focus on putting in place the support and the plan for successfully moving to a dynamic learning culture. 


\section{References}

Argyris, C. (2000). “Double Loop Learning.” Harvard Business Review, SeptemberOctober, 115-125.

Carrillo, P.M., Robinson, H.S., Al-Ghassani, A.M. and Anumba, C.J., (2004).

“Knowledge Management in UK Construction: Strategies, Resources and Barriers.” Project Management Journal, 35(1), 46-56.

Chinowsky, Paul S. and Molenaar, Keith R. (2005). "Learning Organizations in Construction,” Proceedings of the 2005 Construction Research Congress, San Diego, CA, ASCE.

Drucker, Peter F. Post-Capitalist Society. Harper Business, 1993.

Fu, W.K., Drew, D.S. and Lo, H.P. (2002). "The effect of experience on contractors' competitiveness in recurrent bidding.” Construction Management and Economics, 20, 655-666.

Goh, Swee C. (1998). “Toward a Learning Organization: The Strategic Building Blocks.” Advanced Management Journal, 63(2).

Hendricks, P.H.J. and Vriens, D.J. (1999). “Knowledge-based systems and knowledge management: friends or foes?” Information \& Management, 35. 113-125.

Kululanga, G.K., McCaffer, R., Price, A.D.F., and Edum-Fotwe, F. (1999). “Learning mechanisms employed by construction contractors.” Journal of Construction Engineering and Management, 125(4), 215-223. 
Luthans, F., Rubach, M.J., and Marsnik, P. (1995). “Going Beyond Total Quality: The characteristics, techniques, and measures of learning organizations.” The international Journal of Organizational Analysis, 3(1), 24-44.

McGill, Michael E., Slocum, John W. Jr., and Lei, David (1992). “Management Practices in Learning Organizations.” Organizational Dynamics, 21(1), 4-17.

McInerney, C. (2002). "Knowledge management and the dynamic nature of knowledge.” Journal of the American Society for Information Science and Technology, 53(12), 1009-1018.

Messner, J. (2003). “An Architecture for Knowledge management in the AEC Industry.” Proc. Of the 2003 ASCE Construction Research Congress, Molenaar, K. and Chinowsky, P. (eds.), Honolulu, HI, March 2003.

Nevis, E.C., DiBella, A.J., and Gould, Janet M. (1995). “Understanding organizations as learning systems.” Sloan Management Review, 36(2), 73-85.

Nonaka, I. and H. Takeuchi (1995) The Knowledge Creating Company. Oxford University Press, Oxford.

Robinson, H.S., Carrillo, P.M., Anumba, C.J. and Al-Ghassani, A.M. (2004) "Developing a Business case for Knowledge Management: the IMPaKT approach" , Construction Management and Economics , 22(7) , 733-743. Senge, Peter M. (1990). The Fifth Discipline, Currency/Doubleday, New York.

Stata, R. (1989). “Organizational learning - The key to management innovation,” Sloan Management Review, 30(3), 63-74. 


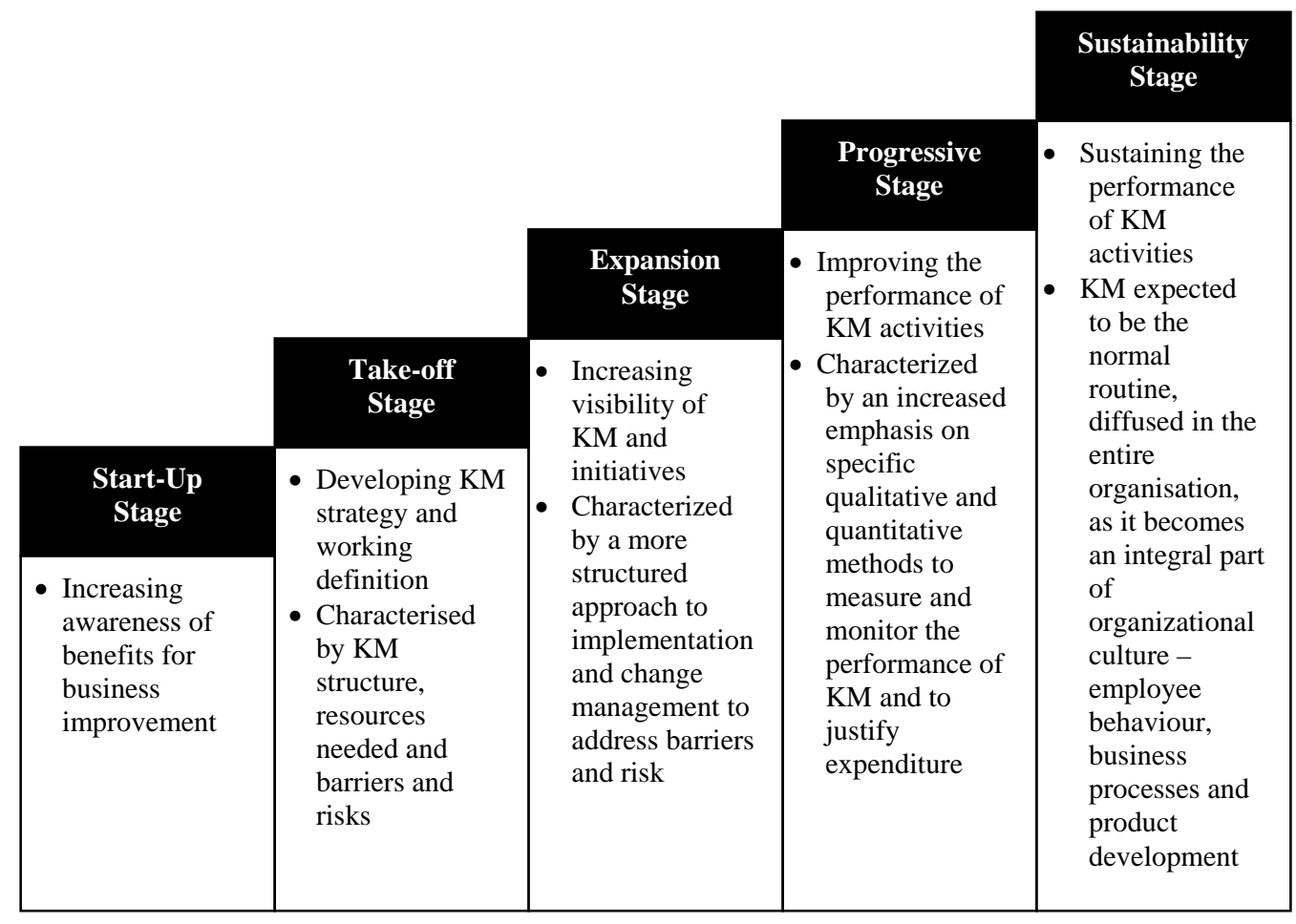

Figure 1: The STEPS Model 


\begin{tabular}{|l|l|l|l|}
\hline \multicolumn{1}{|c|}{ CHARACTERISTICS } & \multicolumn{3}{c|}{ LEARNING ORGANIZATION LEVELS } \\
ORGANIZATION & \multicolumn{1}{c|}{ COMMUNITY } & INDIVIDUAL \\
\hline Leadership & & & \\
\hline Processes and Infrastructure & & & \\
\hline Communication/Collaboration & & & \\
\hline Education & & & \\
\hline Culture & & & \\
\hline
\end{tabular}

Figure 2: The Learning Organization Matrix is comprised of Learning Levels and Characteristics. When characteristics are applied at each level, specific responsibilities are developed for organization employees. 


\section{Case Study Interview Topics}

\section{Organization Overview}

Gain an understanding of the organization and its knowledge management and learning initiatives.

\section{Knowledge Sharing}

What is the organization pursuing to try and increase knowledge sharing within all parts of the organization?

\section{Communities of Practice}

Gain an understanding of the communities of practice in place and what direction the organization is proceeding in this topic.

\section{Leadership Support}

At what levels of management are learning initiatives being supported and what resources are being allocated to the initiatives?

\section{Barriers and Strategies}

What are the barriers that are being encountered during the implementation phases of the initiatives and what strategies are being used to overcome the barriers?

Figure 3: Outline of Case Study Interview Questions 


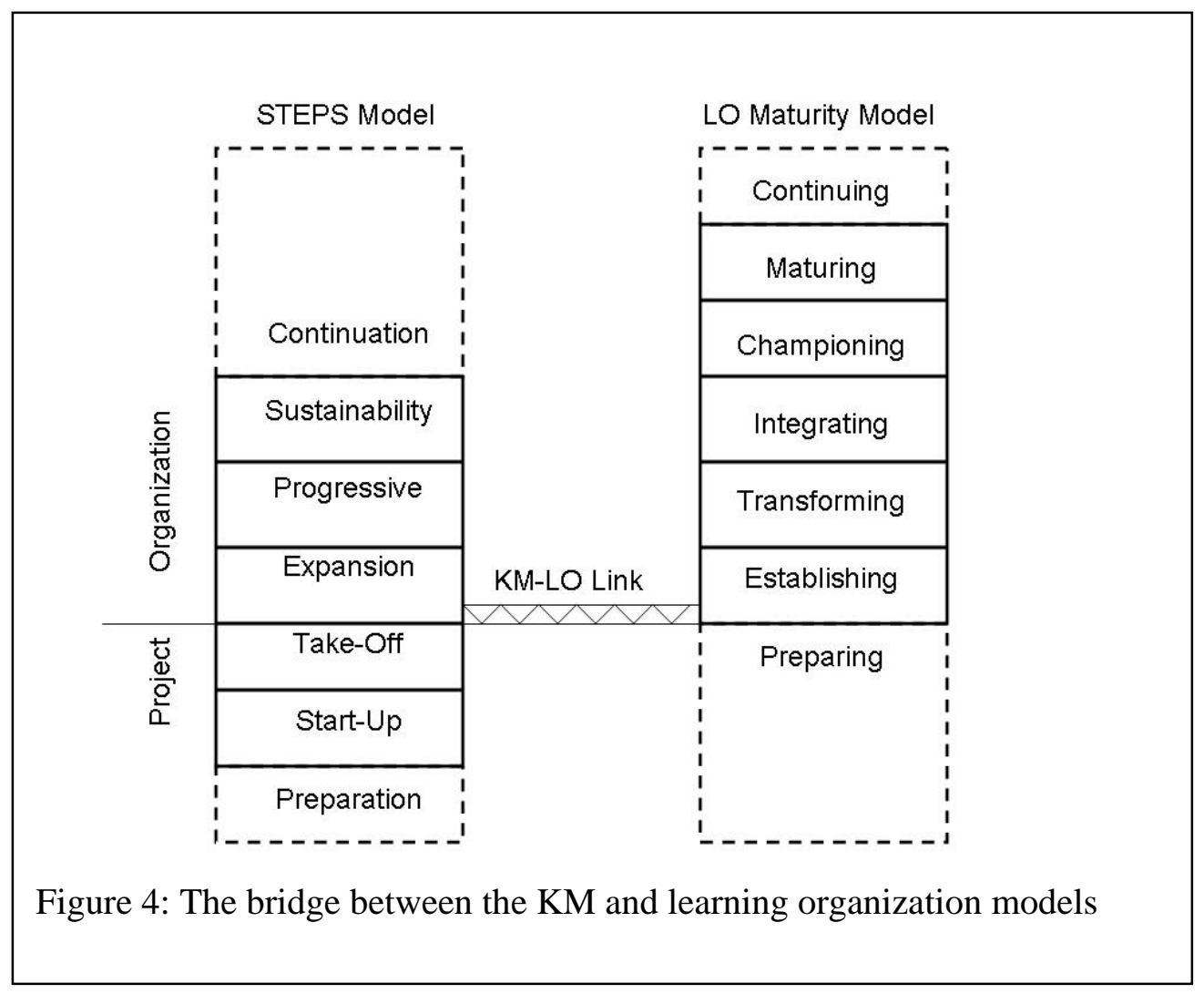

